\title{
BMJ Open Knowledge, training and willingness to perform bystander cardiopulmonary resuscitation among university students in Chongqing, China: a cross- sectional study
}

\author{
Jiani Mao, ${ }^{1}$ Feng Chen, ${ }^{2}$ Dianguo Xing, ${ }^{3}$ Huixian Zhou (D) , ${ }^{1}$ Ling Jia, ${ }^{1}$ \\ Yan Zhang (iD) ${ }^{1}$
}

To cite: Mao J, Chen F, Xing D, et al. Knowledge, training and willingness to perform bystander cardiopulmonary resuscitation among university students in Chongqing, China: a crosssectional study. BMJ Open 2021;11:e046694. doi:10.1136/ bmjopen-2020-046694

- Prepublication history for this paper is available online. To view these files, please visit the journal online (http://dx.doi. org/10.1136/bmjopen-2020046694).

Received 08 November 2020 Accepted 01 June 2021
Check for updates

(C) Author(s) (or their employer(s)) 2021. Re-use permitted under CC BY-NC. No commercial re-use. See rights and permissions. Published by BMJ.

For numbered affiliations see end of article.

Correspondence to

Dr Yan Zhang;

cqmudzy@163.com

\section{ABSTRACT}

Objective To evaluate knowledge of cardiopulmonary resuscitation (CPR) among Chinese college students and their attitude towards participating in CPR training and willingness to perform bystander CPR.

Design A cross-sectional study.

Participants A total of 1128 college students were selected through a multistage stratified random sampling method from 12 universities in Chongqing, China.

Primary and secondary outcomes Primary outcomes included CPR knowledge and willingness to participate in training and perform bystander CPR; secondary outcomes included CPR training experience and obstacles to training and performing CPR.

Results The average score on CPR knowledge was 2.078 ( \pm 1.342$)$. Only $45.5 \%$ of the respondents were willing to participate in CPR training. Women, respondents who were postgraduate or above, with liberal arts as major and with high CPR knowledge level were more willing to participate in CPR training. A total of $47.2 \%$ of the respondents were willing to provide simple assistance, such as checking the consciousness and breathing of the patient and dialling 120 (medical emergency call). Only $34.1 \%$ indicated their willingness to perform bystander CPR on strangers. Perceived behavioural control, behavioural attitudes and subjective norms are positive predictors of willingness to provide bystander CPR.

Conclusions CPR knowledge and training rate were low among Chongqing college students. Willingness to participate in training and perform bystander CPR was also low. Improving legislation, strengthening training programmes, incorporating CPR training into the school curriculum and reshaping the social and public culture of offering timely help to those in need are recommended strategies to improve bystander CPR performance.

\section{INTRODUCTION}

Out-of-hospital cardiac arrest (OHCA) is a major public health problem worldwide. ${ }^{1}$ OHCA kills approximately 540000 people a year in China. ${ }^{2}$ Bystander cardiopulmonary resuscitation (CPR) is an important
Strengths and limitations of this study

- The study applied the theory of planned behaviour to understand willingness to perform bystander cardiopulmonary resuscitation (CPR).

- This is the first large-scale survey of college students in Chongqing, the largest city in Western China, which has more than one million college students.

- Respondents' willingness to perform CPR did not mean they would perform CPR in real situations.

contributing factor for improved OHCA survival. ${ }^{3}{ }^{4}$ Research showed that 30-day survival rates were 2.6 times higher for patients in whom bystander CPR was performed than those with no CPR performed. ${ }^{3}$ However, the rate of bystander CPR in several large-sized and medium-sized cities in China is only $4.5 \%,{ }^{5}$ much lower than in European countries and the USA. ${ }^{367}$

The key component of the theory of planned behaviour (TPB) is the concept of behavioural intention, ${ }^{8}$ which is influenced by the attitude about the likelihood that the behaviour will have the expected outcome and the subjective evaluation of the risks and benefits of that outcome. The TPB states that behavioural achievement depends on both motivation (intention) and ability (behavioural control). It distinguishes between three types of beliefs: behavioural, normative and control. The theory has been widely used in the analysis of health behaviour and behavioural intentions. ${ }^{9-12}$ According to the TPB, intention to perform bystander CPR is the most direct predictor of bystander CPR behaviour. Therefore, in addition to factors such as personal characteristics ${ }^{13}$ and personal experiences, ${ }^{14}$ incorporating the constructs 
of the TPB would better facilitate understanding of the contributing factors for performing bystander CPR.

Having adequate CPR knowledge and skills was a key factor in performing CPR. ${ }^{15}$ Training more people is an effective way to improve bystander CPR. ${ }^{16}{ }^{17}$ However, the rate of CPR training in China is less than $1 \%{ }^{18}$ In 2019, the Chinese government released the 'Healthy China Initiative (2019-2030)' policy, which clearly stated that more than $3 \%$ of the population should be certified in first aid training by 2030. Baldi et $a l^{19}$ showed that students needed less time learning basic life support (BLS) and had better results compared with adults. Popularising bystander CPR knowledge and skills among students is an important way to improve access to bystander CPR. ${ }^{20}$ University students are one of the important groups that can potentially perform bystander CPR and spread CPR knowledge and skills. Except for Tianjin ${ }^{21}$ and Wuhan, ${ }^{22}$ data on CPR training and willingness to perform CPR are still lacking in many other areas of China, especially in Western China.

In this study, we intended to investigate CPR knowledge, current status of CPR training and willingness to perform bystander CPR among university students in Chongqing, Western China. We also attempted to explore the factors that influence CPR training and willingness to perform bystander CPR based on the constructs of the TPB framework to identify the determinants of behavioural intentions of CPR and develop effective interventions.

\section{METHODS}

\section{Study setting and data collection}

Our study was conducted among university students in Chongqing, China from October 2019 to December 2019. Chongqing is one of the four municipalities directly under the jurisdiction of the Chinese Central Government. It has a population of 31.24 million, including 1.04 million university students, making it the city with the largest number of college students in Western China. The city is home to a total of 27 universities, with 13 disciplines and 1150 majors.

We used a multistage random sampling method. First, 12 universities were randomly selected from 27 universities. Second, one male and one female student hostels were randomly selected from every university. Third, 13 dormitories were randomly selected from each student hostel, and all students in the dormitories were asked to complete a questionnaire. All students were briefed on the purpose of the study and filled in the electronic questionnaires by scanning the $\mathrm{QR}$ (quick response) code. All students who participated in the survey gave their informed consent before completing the questionnaire. Each completed questionnaire was assigned a unique anonymous number. Incomplete and contradictory answers were deemed invalid. Based on the results of similar surveys in other studies (the proportion of university students who were willing to perform CPR is approximately $30 \%$ with a margin of error of $3 \%$ ), the required sample size was estimated to be 1106 , assuming $10 \%$ nonresponses and $10 \%$ unqualified answers.

\section{Measures}

Our study used a five-part structured questionnaire.

- Part 1: demographic information, including gender, education and major.

- Part 2: CPR knowledge, including six questions and each question has only one correct answer. One mark was given for the right answer and the maximum total score was six marks.

- Part 3: CPR training, including training experience, willingness and obstacles.

- Part 4: constructs of the TPB. A total of six questions in three dimensions consisting of subjective norms, behavioural attitudes and perceived behavioural control were included. A 5-point Likert scale was used in each question (from $1=$ strongly disagree/strongly unconfident/very few to 5 =strongly agree/strongly confident/great majority). The Cronbach's alpha of the scale was 0.820 .

- Part 5: willingness and obstacles to performing CPR.

\section{Data analysis}

Continuous variables were expressed as mean and SD, and categorical variables were expressed as count and composition ratio. Liberal arts included philosophy, history, law, literature, etc. Sciences included engineering, agriculture, medicine, etc. The total score on CPR knowledge was calculated and divided into high level (score median >2) and low level (score median $\leq 2)$. The $\chi^{2}$ test was used to compare the differences in categorical outcomes between trained and untrained respondents. Logistic regression was used to explore the influencing factors of willingness to participate in CPR training.

The influencing factors of willingness to perform CPR were explored using hierarchical logistic regression. Predicting variables were entered into the model in a specified order to study the extent to which the newly included variables explained the outcome variables. Predicting variables were grouped and entered into the model in the following order:

- In the first block, demographic variables were entered.

- In the second block, knowledge level, training experience and the TPB variables were entered.

The OR and $95 \%$ CI of OR were presented. Statistical analyses were performed using SAS V.9.4. $\mathrm{P}<0.05$ was considered statistically significant.

\section{RESULTS}

A total of 1171 questionnaires were completed. After excluding 43 questionnaires with incomplete or contradictory answers, 1128 questionnaires were included for final analyses. 


\begin{tabular}{|c|c|c|}
\hline & Total (n) & Percentage \\
\hline \multicolumn{3}{|l|}{ Gender } \\
\hline Male & 414 & 36.7 \\
\hline Female & 714 & 63.3 \\
\hline \multicolumn{3}{|l|}{ Education } \\
\hline Lower-grade undergraduate & 509 & 45.1 \\
\hline Upper-grade undergraduate & 565 & 50.1 \\
\hline Postgraduate or above & 54 & 4.8 \\
\hline \multicolumn{3}{|l|}{ Major } \\
\hline Sciences & 583 & 51.7 \\
\hline Liberal arts & 545 & 48.3 \\
\hline \multicolumn{3}{|l|}{ CPR training experience } \\
\hline Yes & 379 & 33.6 \\
\hline No & 749 & 66.4 \\
\hline
\end{tabular}

CPR, cardiopulmonary resuscitation.

\section{Participants}

Of the respondents, $714(63.3 \%)$ were male. Lower-grade undergraduate students (ie, freshmen and sophomores) accounted for $45.1 \%$ and upper-grade undergraduate students (ie, junior and senior) accounted for $50.1 \%$. About half (48.3\%) of the respondents majored in liberal arts (table 1).

\section{CPR knowledge}

The proportion of correct answers on CPR knowledge is shown in table 2. The average score on CPR knowledge was $2.078( \pm 1.342)$, and the median score was 2 . Only $1 \%$ answered all the questions correctly. Only $14.0 \%, 21.5 \%$ and $28.9 \%$ of the respondents knew 'the frequency and depth of chest compressions during CPR', 'the way of judging unconscious' and 'how to determine respiratory arrest', respectively. The average knowledge score of those who received CPR training was $2.430( \pm 1.408)$, which was significantly higher than of those who did not receive CPR training (mean $=1.900, \mathrm{p}<0.001$ ).

\begin{tabular}{|c|c|c|}
\hline CPR knowledge & Total (n) & Percentage \\
\hline How to determine respiratory arrest & 326 & 28.9 \\
\hline How to determine cardiac arrest & 572 & 50.7 \\
\hline Way of judging an unconscious & 243 & 21.5 \\
\hline $\begin{array}{l}\text { Hand position of chest } \\
\text { compressions during CPR }\end{array}$ & 594 & 52.7 \\
\hline $\begin{array}{l}\text { Frequency and depth of chest } \\
\text { compressions during CPR }\end{array}$ & 158 & 14.0 \\
\hline Correct operating sequence of CPR & 451 & 40.0 \\
\hline
\end{tabular}

CPR, cardiopulmonary resuscitation.

\section{Training experience and willingness}

Only $33.6 \%$ of the respondents received CPR training (table 1). No significant difference was observed in CPR training rates according to gender, education and different majors. Among the trained respondents, $46.9 \%$ had a total training duration of less than 2 hours and $28.6 \%$ had a total duration of more than 2 hours but less than 4 hours. A total of $44.9 \%$ of the respondents had only received lecture-based training and $55.1 \%$ had received lecture-based and hands-on training. Schools $(76.8 \%)$ were the primary places where respondents participated in CPR training.

Only $45.5 \%$ of the respondents were willing to participate in CPR training. In the logistic regression model, women, respondents who were postgraduate or above, with liberal arts as major and high CPR knowledge level were more willing to participate in CPR training (table 3).

Respondents wished to participate in training through radio $(62.8 \%)$, internet $(62.5 \%)$, lectures $(61.8 \%)$ and medical personnel $(59.0 \%)$, and the most desired location for CPR training was schools $(80.9 \%)$, followed by hospitals $(61.5 \%)$.

The biggest obstacle to participating in CPR training was the difficulty of access to learning $(61.1 \%)$, and the proportion was even higher $(65.2 \%)$ for untrained respondents. More untrained respondents considered lack of time $(39.8 \%)$ and awareness towards the importance of CPR (27.8\%) as barriers to participating in CPR training than trained participants. However, more trained respondents $(50.9 \%)$ considered lack of opportunities to use the learnt skills as a barrier (table 4).

\section{Results for variables in the TPB}

The results for the TPB variables are presented in table 5 . The average scores for behavioural attitude, subjective norms and perceived behavioural control were 3.242 $( \pm 1.288), 3.140( \pm 1.000)$ and $2.848( \pm 1.019)$, respectively. Only $23.1 \%$ of the respondents had confidence in CPR. Only $32.2 \%$ of the respondents thought that most people would perform bystander CPR on strangers, and $49.5 \%$ believed that bystander CPR was valuable and could give them a sense of social value.

\section{Willingness to perform bystander CPR}

Only $47.2 \%$ of the respondents were willing to provide some simple assistance, such as checking consciousness and breathing and dialling 120 (medical emergency call). However, only $34.1 \%$ would like to perform bystander CPR on strangers. In model 1 of the logistic regression, women, respondents with liberal arts as major, untrained respondents and those with high level of knowledge were more willing to check consciousness and breathing and dial 120. Model 2 showed no significant difference in willingness to perform bystander CPR between different genders and majors. Model 3 showed that perceived behavioural control, behavioural attitude and subjective norms were significant positive predictors of willingness to provide bystander CPR (table 6 ). 
Table 3 Logistic regression analysis: influence of demographic variables and CPR knowledge on willingness to participate in CPR training

\begin{tabular}{|c|c|c|c|}
\hline \multirow[b]{2}{*}{ Variables } & \multicolumn{3}{|c|}{ Willingness to participate in CPR training } \\
\hline & Willing to participate, $\mathrm{n}(\%)$ & OR (95\% Cl) & $P$ value \\
\hline \multicolumn{4}{|l|}{ Gender } \\
\hline Female & $356(49.9)$ & $1.493(1.149$ to 1.941$)$ & 0.003 \\
\hline \multicolumn{4}{|l|}{ Education } \\
\hline Postgraduate or above & $40(74.1)$ & $2.508(1.319$ to 4.768$)$ & 0.005 \\
\hline \multicolumn{4}{|l|}{ Major } \\
\hline Sciences & $222(38.1)$ & 1.0 & \\
\hline Liberal arts & $291(53.4)$ & 1.755 (1.365 to 2.257$)$ & 0.000 \\
\hline
\end{tabular}

CPR, cardiopulmonary resuscitation.

Lack of confidence $(66.6 \%)$, fear of causing secondary damage to patients $(56.4 \%)$ and fear of legal liability $(37.4 \%)$ were the main obstacles to performing bystander CPR among the respondents. Compared with trained respondents, untrained respondents were more likely to lack confidence $(69.6 \%)$ and fear causing secondary damage $(59.8 \%)$ (table 7 ).

\section{DISCUSSION}

The results of our study showed that respondents lacked CPR knowledge, with an average CPR knowledge score of 2.078 (out of a total score of 6). Lack of formal and repeated training was probably the main reason for the low CPR knowledge. The results showed that only $33.6 \%$ of the respondents had taken part in CPR training, much lower than Norway (89\%). ${ }^{23}$ Only $45.5 \%$ of the college students were willing to attend CPR training, far lower than New Zealand $(63 \%),{ }^{24}$ Belgium $(77.0 \%)^{25}$ and
Wuhan, a city in Central China $(88.3 \%) .{ }^{26}$ These findings may be related to respondents' lack of CPR knowledge and awareness of the importance of training and performing bystander CPR. Our study showed that approximately a quarter of the respondents reported being unaware of the importance of bystander CPR, regardless of whether they had attended training or not. It is necessary to emphasise the importance of bystander CPR in saving patients' lives through successful examples and comparative data, rather than just teaching skills. ${ }^{27}$

The low CPR knowledge and training rate and unawareness of the importance of bystander CPR may be related to perceived barriers to training. A total of $61.1 \%$ of the respondents reported difficulties in accessing training, and $42.6 \%$ worried about the disconnect between theory and practice during training and the unsatisfactory results. In China, public CPR training has always relied mainly on social groups such as the Red Cross. Due to manpower

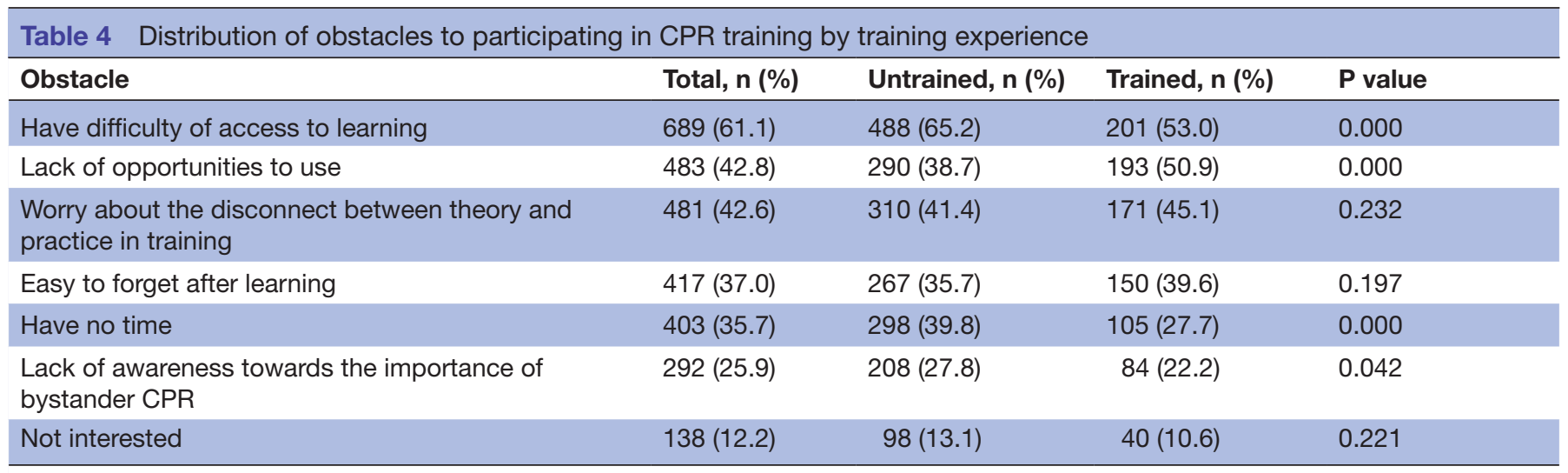

CPR, cardiopulmonary resuscitation. 
Table 5 Variables in the theory of planned behaviour

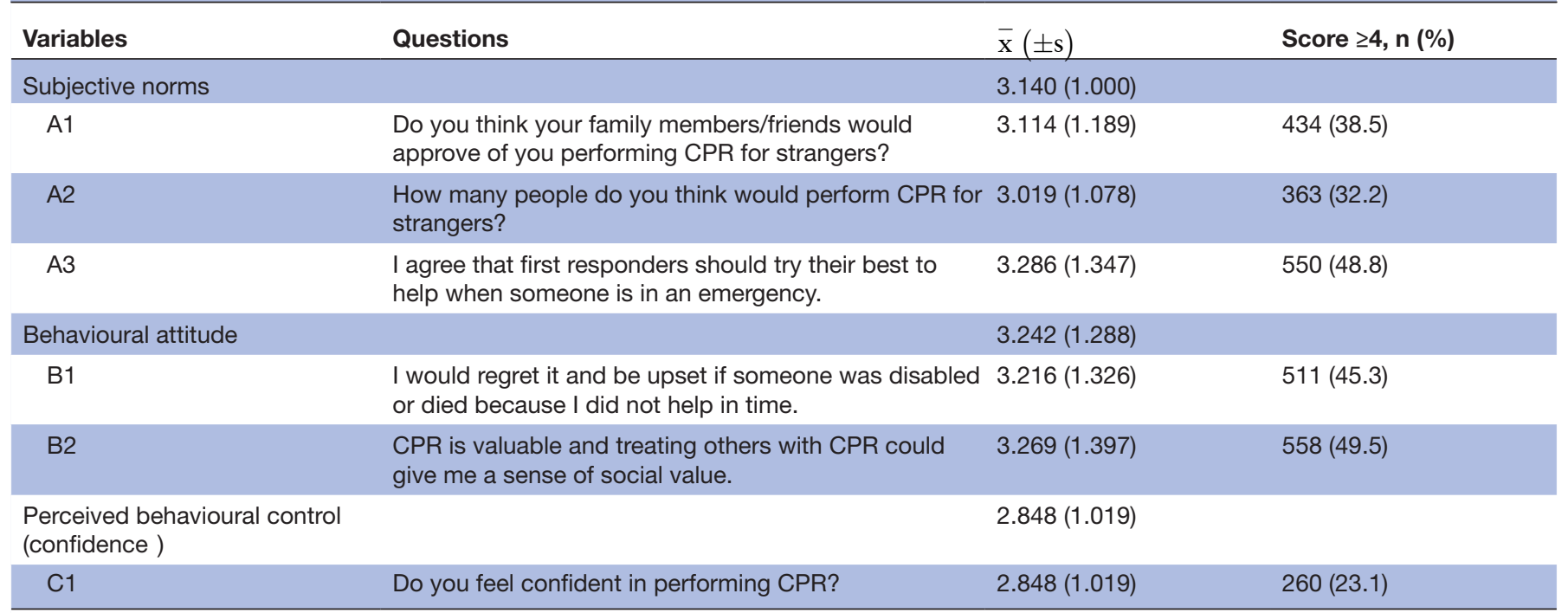

CPR, cardiopulmonary resuscitation.

and funding constraints, lecture-based training was the most common method. ${ }^{28}$ In 2018, the Chongqing Municipal Government officially launched the Public Health Emergency Skills Improvement Initiative, which identified CPR training as a priority. However, due to the short time period that the project has been running, its social impact remains insufficient. Our subgroup analysis revealed that senior undergraduate students were more reluctant to participate in CPR training, which may be related to the pressures they face in terms of graduation, seeking employment and further education. These pressures can make it difficult for them to focus on other subjects, especially when there are many obstacles to that subject. ${ }^{29}$

Less than half of the respondents were willing to provide simple assistance, such as checking consciousness and breathing and dialling 120 . Only $34.1 \%$ of the respondents were willing to perform CPR on strangers, which was lower than Belgium $(68.0 \%)^{25}$ and Germany $(72.3 \%){ }^{30}$ but was similar to Tianjin $(44.4 \%) .{ }^{14}$ This finding may be related to the social environment and culture of China. First, unlike other countries where emergency medical services can be provided by firemen ${ }^{31}$ or emergency lifesaving technicians, ${ }^{32}$ in China prehospital emergency care has always been provided by doctors and nurses with medical qualifications. ${ }^{33}$ The idea that patients should be examined and treated by medical personnel is so deeply rooted that a significant proportion of the respondents may not be aware of the importance of bystander first aid and their corresponding social responsibility. ${ }^{34}$ The results with regard to the behavioural attitudes in the TPB showed that less than half of the respondents believed that saving others through bystander CPR could give them a sense of social value. Only $45.3 \%$ of the respondents believed that they would regret and be upset if their failure to save others in time had led to their disability or death. Second, the mouth-to-mouth practice could influence the willingness to perform bystander CPR on a stranger. ${ }^{35}$ Mouth-to-mouth may be considered unhygienic and risks spread of disease. Our results showed that $15.8 \%$ of the respondents were afraid of being infected with a disease as a result of performing CPR. In a traditional Chinese culture, kissing others in public is impolite and performing mouth-to-mouth on a stranger can be embarrassing. ${ }^{14}{ }^{36}$ Finally, in 2017, the provision of the 'Good Man Law', which was included in the General Principles of the Civil Law of China, is not yet sufficiently influential in the society. There is also a lack of systematic provisions to exempt strangers from the liability of administering first aid, thereby making it less operable. A total of $37.4 \%$ of the respondents reported concerns about legal disputes that arose from providing help, lower than the general Chinese public $(53.2 \%),{ }^{37}$ but higher than the USA $(4.1 \%)^{38}$ and Scotland (8.0\%). ${ }^{13}$ Although helping others has always been a traditional Chinese virtue, such a tradition has been weakened. The reasons include the rapid urbanisation shifting relationships between acquaintances to relationships between strangers, ${ }^{39}$ and the absence of relevant laws over a long time had led to many high profile media cases of 'saving a person but being blackmailed'. ${ }^{40}$ Despite the recent progress, there is still a long way to go for China to develop proper social climate and public habits for providing timely rescues to those in need, such as performing CPR on strangers. The results of the survey also showed that less than $40 \%$ of the respondents believed that their family and friends would support them in performing bystander CPR on a stranger. Despite scoring low, subjective norms had a positive effect on willingness to perform CPR.

Our analyses also found that women and liberal arts students, in addition to being more willing to participate in CPR training, were also more willing to provide simple 


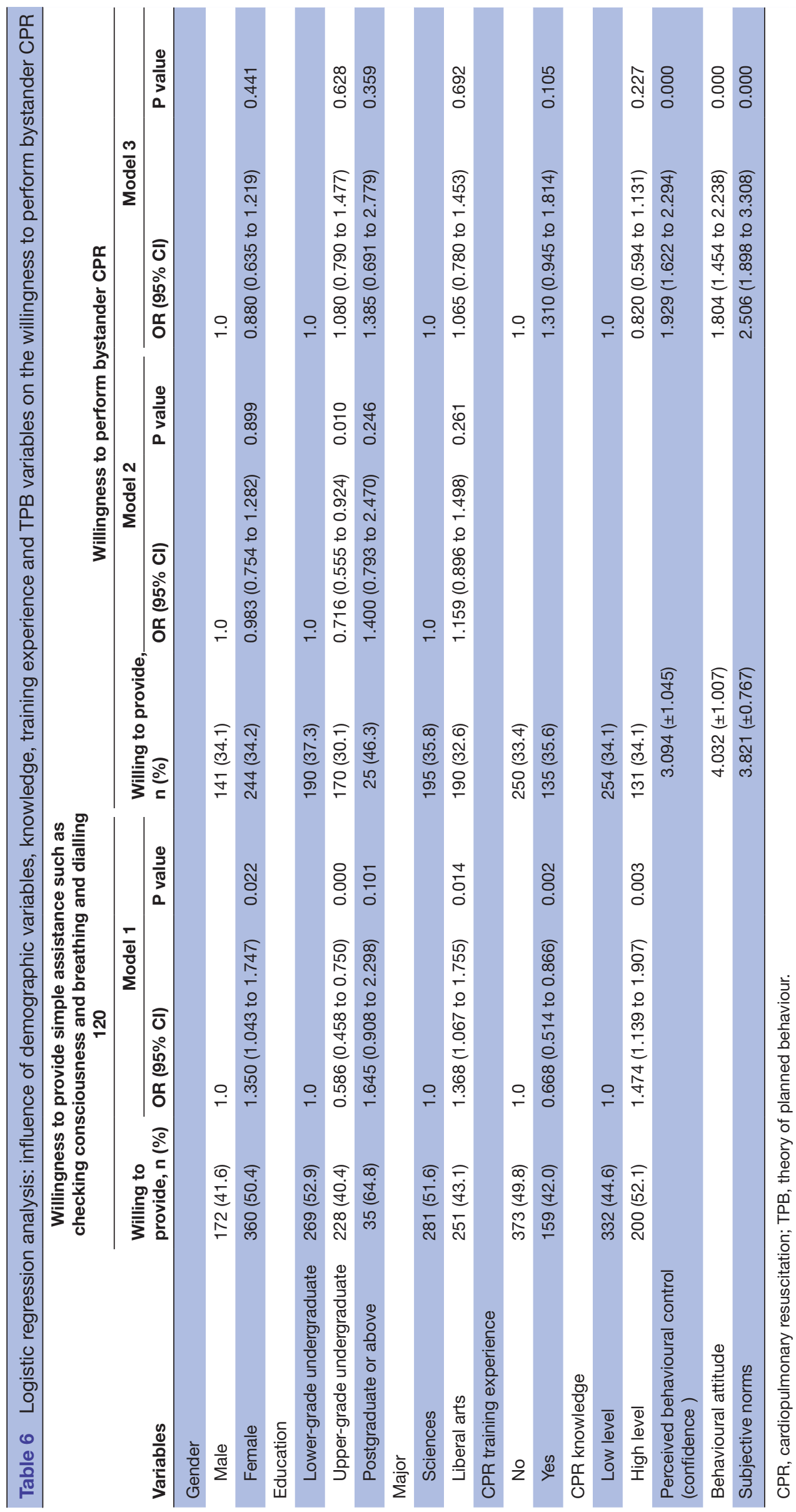


Table 7 Distribution of obstacles to performing CPR by training experience

\begin{tabular}{|c|c|c|c|c|}
\hline Obstacles & Total, n (\%) & Untrained, n (\%) & Trained, n (\%) & P value \\
\hline Lack confidence & $751(66.6)$ & $521(69.6)$ & $230(60.7)$ & 0.003 \\
\hline Fear of causing secondary damage to patients & $636(56.4)$ & $448(59.8)$ & $188(49.6)$ & 0.001 \\
\hline Fear of infectious diseases & $178(15.8)$ & $116(15.5)$ & $62(16.4)$ & 0.705 \\
\hline Fear of public opinion & $148(13.1)$ & $102(13.6)$ & $46(12.1)$ & 0.487 \\
\hline
\end{tabular}

CPR, cardiopulmonary resuscitation.

assistance with consciousness and breath checks and dialling 120, which may be related to the fact that women and arts students are more emotional and empathic. ${ }^{414}$ Although science students include some medical students, evidence shows that CPR knowledge among medical students is still low. ${ }^{43}$ A significant proportion of medical students did not attend BLS training during their study period. ${ }^{43}$ A recent European Resuscitation Council guidance note strongly promotes strengthening CPR training for medical undergraduates. ${ }^{44}$ Training experience and high level of knowledge were not influencing factors in the willingness to perform bystander CPR. Respondents who attended training were more unwilling to provide simple help than non-participants; such findings were unlike other relevant studies, ${ }^{1516}$ but similar to the results of the study by Huang $e t a l .{ }^{26}$ Such findings may be related to the short duration and poor quality of training. Of those who had attended CPR training, $75.5 \%$ reported that it lasted less than 4 hours, and nearly half reported having attended only lecture-based training with no hands-on experience. There is also a need to further explore whether poor CPR knowledge after training had led to increased concerns about the possible negative consequences of providing help and reduced willingness to help. The disappointing CPR training results may also be due to lack of emphasis on increasing willingness to perform bystander CPR. Our results showed that behavioural attitudes, subjective norms and perceived behavioural control positively affected willingness to perform bystander CPR. However, respondents scored low on all three dimensions. Perceived behavioural control scored the lowest, with respondents generally lacking confidence in performing bystander CPR $(66.6 \%$ citing a lack of confidence in their first aid ability as a barrier to performing bystander CPR and $56.4 \%$ feared causing secondary injury to the injured person). Therefore, in addition to improving participants' knowledge and skills, future training should also focus on improving their self-confidence in performing bystander CPR. Measures may be included, such as providing discussions about worries and fears in the course, training skills in overcoming and reducing fears and improving confidence, ${ }^{45}$ and combining media publicity and education campaign to enhance positive behavioural attitudes and subjective norms.

In summary, we suggest that the Chinese government and social organisations work together to develop a strategic plan to improve bystander CPR performance, focusing on the following areas. First is to improve relevant legislation and close legal loopholes to protect the legitimate rights and interests of rescuers by introducing more detailed and operational legal provisions on the exemption from liability for public first aid. Second is to create a social atmosphere and culture of encouraging the public to offer help to those in need and reinforcing the positive image of performing CPR through various ways of mass media and internet publicity and education campaign. Third is to encourage participation and standardising training materials by developing standardised online and offline training courses tailored to the population's characteristics, emphasising training to overcome panic and boost confidence, and incorporating CPR training and retraining into the school curriculum in a planned manner. Finally, reform emergency medical rescue dispatch agencies to enhance its functions in instructing bystanders on performing CPR by video or phone through an app to boost the confidence of rescuers and reduce panic during implementation. All these suggestions are in line with the 2020 International Consensus on Cardiopulmonary Resuscitation and Emergency Cardiovascular Care Science with Treatment Recommendations. ${ }^{46}$

\section{Limitations}

Our study has several limitations. First, this research was carried out in Chongqing and targeted college students. Therefore, the results cannot fully represent the situation in China and other settings. Second, at present, no existing validated planning behaviour theory scale is available in the field of CPR and the scale we have developed may still have inadequacies. Third, our research was conducted through a questionnaire survey and the behavioural intentions were measured in a hypothetical scenario. Thus, the positive behavioural intentions do not necessarily mean actual occurrence of the behaviours in the real world. Future research 
should focus on the actual CPR performance and its determinants.

\section{CONCLUSIONS}

Knowledge level, training rates and willingness to participate in CPR training were low among college students of Chongqing, China. Behavioural attitudes, subjective norms and perceived behavioural control were positively related to willingness to perform bystander CPR, which was also low among college students. Further improvement strategies such as sound legislation, enhanced training, inclusion of CPR in the school curriculum and reshaping the social and public culture of offering timely help to those in need are recommended.

\section{Author affiliations}

${ }^{1}$ School of Public Health and Management, Research Center for Medicine and Social Development, Innovation Center for Social Risk Governance in Health, Chongqing Medical University, Yuzhong District, Chongqing, China

${ }^{2}$ Department of Pre-Hospital Emergency, Chongqing Emergency Medical Centre, Chongqing University Central Hospital, Yuzhong District, Chongqing, China ${ }^{3}$ Office of Health Emergency, Chongqing Municipal Health Commission, Yubei District, Chongqing, China

Acknowledgements The authors would like to express their gratitude to every university and participant in this survey.

Contributors YZ, JM, HZ, DX and FC designed the survey. JM, HZ and LJ conducted the survey and collected the data. $Y Z$ and JM organised and analysed the data. YZ and JM wrote the manuscript. FC and DX provided feedback to the drafts of the manuscript. All authors reviewed the content of the manuscript and approved the final version of the manuscript.

Funding This work was supported by the Chongqing Federation of Social Science Circles (grant number 2019YBGL057) and the Medical Research Project of Chongqing Science and Technology Commission united with Chongqing Municipal Health Commission (grant number 2019ZLXM002).

Competing interests None declared.

Patient consent for publication Obtained.

Ethics approval All participating students gave their verbal informed consent before completing the questionnaire. This study was approved by the Ethics Committee of Chongqing Medical University (date approved: 8 October 2019).

Provenance and peer review Not commissioned; externally peer reviewed.

Data availability statement № additional data available.

Open access This is an open access article distributed in accordance with the Creative Commons Attribution Non Commercial (CC BY-NC 4.0) license, which permits others to distribute, remix, adapt, build upon this work non-commercially, and license their derivative works on different terms, provided the original work is properly cited, appropriate credit is given, any changes made indicated, and the use is non-commercial. See: http://creativecommons.org/licenses/by-nc/4.0/.

\section{ORCID iDs}

Huixian Zhou http://orcid.org/0000-0003-3202-4851

Yan Zhang http://orcid.org/0000-0003-3533-2809

\section{REFERENCES}

1 Kiguchi T, Okubo M, Nishiyama C, et al. Out-Of-Hospital cardiac arrest across the world: first report from the International liaison Committee on resuscitation (ILCOR). Resuscitation 2020;152:39-49.

2 ST H, Gao RL, Wang Z. Report on cardiovascular diseases in China 2018. 149. Encyclopedia of China Publishing House, 2019. https:// www.nccd.org.cn/News/Columns/Index/1089

3 Hasselqvist-Ax I, Riva G, Herlitz J, et al. Early cardiopulmonary resuscitation in out-of-hospital cardiac arrest. $N$ Engl J Med 2015;372:2307-15.
4 Travers AH, Perkins GD, Berg RA, et al. Part 3: adult basic life support and automated external defibrillation: 2015 international consensus on cardiopulmonary resuscitation and emergency cardiovascular care science with treatment recommendations. Circulation 2015;132:S51-83.

5 Xu F, Zhang Y, Chen Y. Cardiopulmonary resuscitation training in China: current situation and future development. JAMA Cardiol 2017;2:469-70.

6 Malta Hansen C, Kragholm K, Pearson DA, et al. Association of bystander and First-Responder intervention with survival after out-of-hospital cardiac arrest in North Carolina, 2010-2013. JAMA 2015;314:255--64.

7 Brinkrolf P, Bohn A, Lukas R-P, et al. Senior citizens as rescuers: is reduced knowledge the reason for omitted lay-resuscitationattempts? results from a representative survey with 2004 interviews. PLoS One 2017;12:e0178938.

8 Fishbein M, Ajzen I, Belief A. Contemporary Sociology. In: Belief, attitude, intention, and behavior: an introduction to theory and research. 6, 1977.

9 Gantt CJ. The theory of planned behavior and postpartum smoking relapse. J Nursing Scholarship 2001;33:337-41.

10 Kuther TL. Rational decision perspectives on alcohol consumption by youth. revising the theory of planned behavior. Addict Behav 2002;27:35-47.

11 Chung LMY, Fong SSM. Predicting actual weight loss: a review of the determinants according to the theory of planned behaviour. Health Psychol Open 2015;2:2055102914567972:205510291456797.

12 Conner M, Norman P, Bell R. The theory of planned behavior and healthy eating. Health Psychol 2002;21:194-201.

13 Dobbie F, MacKintosh AM, Clegg G, et al. Attitudes towards bystander cardiopulmonary resuscitation: results from a crosssectional general population survey. PLoS One 2018;13:e0193391.

14 Lu C, Jin Y, Meng F, et al. An exploration of attitudes toward bystander cardiopulmonary resuscitation in university students in Tianjin, China: a survey. Int Emerg Nurs 2016;24:28-34.

15 Nordberg P, Hollenberg J, Herlitz J, et al. Aspects on the increase in bystander CPR in Sweden and its association with outcome. Resuscitation 2009;80:329-33.

16 Tanigawa K, Iwami T, Nishiyama C, et al. Are trained individuals more likely to perform bystander CPR? an observational study. Resuscitation 2011;82:523-8.

17 Bhanji F, Donoghue AJ, Wolff MS, et al. Part 14: education: 2015 American heart association guidelines update for cardiopulmonary resuscitation and emergency cardiovascular care. Circulation 2015;132:S561-73.

18 Cardiopulmonary Resuscitation Specialized Committee of Chinese Research Hospital Association, the Science Popularization Branch of the Chinese Medical Association, Wang L, et al. [2018 National consensus on cardiopulmonary resuscitation training in China]. Zhonghua Wei Zhong Bing Ji Jiu Yi Xue 2018;30:385-400.

19 Baldi E, Bertaia D, Contri E. School children learn BLS better and in less time than adults. Resuscitation 2015;88:e15-16.

20 Cave DM, Aufderheide TP, Beeson J, et al. Importance and implementation of training in cardiopulmonary resuscitation and automated external defibrillation in schools: a science Advisory from the American heart association. Circulation 2011;123:691-706.

21 Lu C, Jin Y-H, Shi X-T, et al. Factors influencing Chinese university students' willingness to performing bystander cardiopulmonary resuscitation. Int Emerg Nurs 2017;32:3-8.

22 Chen Z-Q, Zhao Y, Lu Z-H, et al. Awareness and attitudes of Chinese students towards cardiopulmonary resuscitation. Emerg Med $\mathrm{J}$ 2010;27:907-10.

23 Kanstad BK, Nilsen SA, Fredriksen K. Cpr knowledge and attitude to performing bystander CPR among secondary school students in Norway. Resuscitation 2011;82:1053-9.

24 Parnell MM, Pearson J, Galletly DC, et al. Knowledge of and attitudes towards resuscitation in New Zealand high-school students. Emerg Med J 2006;23:899-902.

25 De Smedt L, Depuydt C, Vekeman E, et al. Awareness and willingness to perform CPR: a survey amongst Flemish schoolchildren, teachers and principals. Acta Clin Belg 2019;74:297-316.

26 Huang Q, Hu C, Mao J. Are Chinese students willing to learn and perform bystander cardiopulmonary resuscitation? J Emerg Med 2016;51:712-20.

27 Qi L, Jiang HY, Zhou F. The role of "tailor-made" positive and negative cases combined with teaching model training in residents' first aid knowledge and skills training. Chin J Emerg Resusc Dis Med 2020;15:989-91. 
28 Ling CR, Liu YT, Deng QY. Current situation and demand analysis of the public's knowledge and skills of self-help and mutual rescue. Chin J Integr Trad Med Inten Crit Care 2019;26:719-22.

29 Sun ZF. Analysis and countermeasure of college students' psychological pressure in employment. Edu Vocat 2016;15:59-61.

30 Wanke S, Strack M, Dählmann C, et al. [Defi catches on : Implementation of a curriculum for resuscitation in secondary schools]. Wien Med Wochenschr 2018;168:99-107.

31 Van Gelder CM, Frantz R, Bogucki S. Emergency medical services in Connecticut. Prehosp Emerg Care 2005;9:219-26.

32 Tanigawa K, Tanaka K. Emergency medical service systems in Japan: past, present, and future. Resuscitation 2006;69:365-70.

33 Cai ZB. Present situation and prospect of pre-hospital emergency treatment at home and abroad. Chin J Emerg Med 2010;07:775-7.

34 Kang JH, XX X, Cao J. Discussion on the importance of first aid knowledge training for community citizens. Contin Med Educ 2016;30:163-4.

35 Baldi E, Bertaia D, Savastano S. Mouth-To-Mouth: an obstacle to cardiopulmonary resuscitation for lay-rescuers. Resuscitation 2014;85:e195-6.

36 Jia QH. The cultural history of kiss in modern China. Acad Month 2019;51:152-78.

37 Chen M, Wang Y, Li X, et al. Public knowledge and attitudes towards bystander cardiopulmonary resuscitation in China. Biomed Res Int 2017;2017:1-7.

38 Bogle B, Mehrotra S, Chiampas G, et al. Assessment of knowledge and attitudes regarding automated external defibrillators and cardiopulmonary resuscitation among American university students. Emerg Med J 2013;30:837-41.

39 Wang XZ. "The stranger" : from the other to the new order. Zhejiang Acad J 2019;02:177-84.

40 Cao CS. In what sense does "helping the fallen old" become an ethical challenge. J Inn Mongolia Univ 2017;49:61-6.

41 Pen X, SH M, XM L. Study on the cognitive styles and gender difference of normal university students. Chin J Health Psychol 2006;14:299-301.

42 CP L. Relationship between thinking style and coping style in university students. In: Psychological development and education, 2008: 56-60.

43 Baldi E, Contri E, Bailoni A, et al. Final-year medical students' knowledge of cardiac arrest and CPR: we must do more! Int J Cardiol 2019;296:76-80.

44 Baldi E, Savastano S, Contri E, et al. Mandatory cardiopulmonary resuscitation competencies for undergraduate healthcare students in Europe: a European resuscitation Council guidance note. Eur J Anaesthesiol 2020;37:839-41.

45 Ren ZK, QZ W, Zhang QD. Application of psychological intervention in cardiopulmonary resuscitation training. J Clin Psychosom Dis 2016;22:5-6.

46 Greif R, Bhanji F, Bigham BL, et al. Education, implementation, and teams: 2020 international consensus on cardiopulmonary resuscitation and emergency cardiovascular care science with treatment recommendations. Circulation 2020;142:S222-83. 